\title{
Attenuation of enoyl coenzyme A hydratase 1 expression in colorectal cancer cells using small interfering RNA inhibits cell proliferation and migration
}

\author{
QING-MEI ZHAO $^{1 *}$, FEI KUANG ${ }^{2 *}$, HAN WU $^{3}$ and YU-HAO ZHANG ${ }^{3}$ \\ ${ }^{1}$ Department of Oncology, Sichuan Mianyang 404 Hospital, Mianyang, Sichuan 621000; \\ ${ }^{2}$ Department of General Surgery, Changhai Hospital of The Second Military Medical University, Shanghai 200433; \\ ${ }^{3}$ Department of General Surgery, The $1745^{\text {th }}$ Hospital of the PLA, Zhangzhou, Fujian 562001, P.R. China
}

Received March 23, 2014; Accepted January 29, 2015

DOI: $10.3892 / \mathrm{mmr} .2015 .3418$

\begin{abstract}
Colorectal cancer is one of the most commonly diagnosed types of cancer and is a leading cause of cancer-associated mortality worldwide. Short chain enoyl coenzyme A hydratase 1 (ECHS1) is an important gene involved in the mitochondrial fatty acid $\beta$-oxidation pathway. In addition, ECHS1 has been implicated in a variety of cancers, including breast, prostate, colon and liver cancer. The aim of the present study was to examine the expression of ECHS1 in the human HCT-8 colorectal cancer cell line. The results showed that ECHS1 expression was significantly increased in poorly-differentiated cells compared with that in well-differentiated cells. In order to further investigate the functions of ECHS1 in colorectal cancer cells, a stably transfected HCT-8 cell line expressing small interfering (si) RNA targeting the ECHS1 gene was established. The expression of the ECHS1 siRNA was found to reduce ECHS1 protein levels in ECHS1-silenced cells by $>40 \%$. Cell proliferation and cell migration of the siECHS1 cells were characterized using Cell Counting Kit- 8 and Transwell assays, respectively, the results of which showed that the constitutive knockdown of the ECSH1 gene in HCT-8 cells significantly inhibited cell proliferation and migration. Furthermore, decreased levels of Akt and glycogen synthase kinase (GSK) $3 \beta$ phosphorylation were observed in ECHS1-silenced HCT-8 cells compared with that of parental or pU6 empty vector-transfected cells. In conclusion, the results of the present study suggested that ECHS1 may have an important role in colorectal cancer
\end{abstract}

Correspondence to: Dr Fei Kuang, Department of General Surgery, Changhai Hospital of The Second Military Medical University, 168 Changhai Road, Shanghai 200433, P.R. China

E-mail: kuangfei1980@163.com

*Contributed equally

Key words: enoyl coenzyme A hydratase 1, colorectal cancer, cell proliferation, cell migration cell proliferation and migration via activation of Akt- and GSK3 $\beta$-associated signaling pathways.

\section{Introduction}

Colorectal cancer is one of the most commonly diagnosed types of cancer and is a leading cause of cancer-associated mortality worldwide, particularly among populations in Eastern Asia, Eastern Europe and South America $(1,2)$. Risk factors for colorectal cancer include infection, cigarette smoking, alcohol consumption, diet and genetic variations (3). Although certain progress has been made in the treatment of colorectal cancer, the early diagnosis and long-term survival rates remain low (4); in addition, colorectal cancer was reported to have the second highest mortality rate of all cancer types (5). Therefore, it is essential to gain mechanistic insights into the oncogenesis of colorectal cancer and to identify novel molecular targets for the development of effective therapeutic agents for this highly lethal disease.

The human short chain enoyl coenzyme A hydratase 1 (ECHS1) gene, located at chromosome 10q26.2-q26.3, is a 290 amino acid enzyme which is known to have an essential role in the second step of the mitochondrial fatty acid oxidation pathway (6). ECHS1 is highly conserved among different species and is homologous to other hydrolases and isomerases (7). ECHS1 expression levels were reported to be downregulated in a non-alcoholic fatty liver disease model as well as in patients with hepatic simple steatosis (8). Previous studies have implicated the involvement of ECHS1 in numerous types of cancers, including breast, prostate, colon and liver cancer $(9,10)$. In addition, ECHS1 has been identified as a novel interacting protein of signal transducer and activator of transcription 3 (STAT3) (11). ECHS1 binding was found to specifically inhibit STAT3 activity through repressing STAT3 phosphorylation, thus downregulating the expression of STAT3 target genes (11). Proteomic analysis and an in vitro study revealed that the phosphoinositide 3 kinase (PI3K)-Akt and STAT3 signaling pathways were functionally linked in cancer (12). These findings therefore suggested the possible function of ECHS1 in colorectal cancer. 
The present study aimed to investigate the roles of ECHS1 in colorectal cancer development and progression in order to elucidate a potential molecular biomarker or target for the diagnosis or treatment of colorectal cancer. The expression levels of the ECHS1 gene were determined in the HCT-8 cell line and a stably transfected HCT- 8 cell line was established, in which ECHS1 expression was knocked down using RNA interference technology. These cells were then used to explore the effect of ECHS1 attenuation on cell proliferation, cell migration and the activation of Akt and glycogen synthase kinase (GSK) $3 \beta$ proteins.

\section{Materials and methods}

Cell lines and culture conditions. The human colorectal cancer cell line HCT-8 was purchased from the Cell Bank of the Shanghai Institute of Biochemistry and Cell Biology, Chinese Academy of Sciences (Shanghai, China). HCT-8 cells were cultured in RPMI 1640 media (Invitrogen Life Technologies, Carlsbad, CA, USA) supplemented with $10 \%$ fetal calf serum (Abcam, Cambridge, UK). ECHS1 small interfering (si)RNA vector or empty vector (Santa Cruz Biotechnology, Inc., Dallas, TX, USA) stably transfected cell lines were grown in Dulbecco's modified Eagle's medium (DMEM; Invitrogen Life Technologies) supplemented with $10 \%$ fetal bovine serum [FBS; Shanghai Pharmaecuetical (Group) Co., Ltd., Shanghai, China] and $1 \mu \mathrm{g} / \mathrm{ml}$ puromycin (Invitrogen Life Technologies). All cells were cultured in an incubator at $37^{\circ} \mathrm{C}$ in a $5 \% \mathrm{CO}_{2}$ atmosphere and cell passage was performed using trypsinization with $0.02 \%$ EDTA/0.25\% trypsin (Abcam) (1:1).

Construction and screening of ECHS1-targeting siRNA vectors. The pU6 siRNA expression vector was provided by the Anhui Medical Center laboratory (Anhui, China) and was used to express siRNA targeting the ECHS1 gene (pU6-siECHS1). The pU6 vector expressed the sense- and antisense-strands of the siRNA in tandem under the control of the U6 promoter.

A series of siRNA expression vectors were produced by inserting antisense sequence expression cassettes into sense sequence expression vectors. Sequences inserted immediately downstream of U6 promoter were as follows: siRNA1 sense, 5'-GCUAUGAAACGAUAUGGGCUU-3' and antisense, 5'-GCCCAUAUCGUUUCAUAGCUU-3'; siRNA2 sense, 5'-GUUCGUCACAUCUCAUCUACUU-3' and antisense, 5'-GUAGAUGAGAUGUGACGAAUU-3'.

ECHS1 siRNA stably transfected cell lines. In order to establish ECHS1 siRNA-expressing stably transfected cell lines, HCT- 8 cells were seeded in $60-\mathrm{mm}$ culture dishes and cultured with complete media without antibiotics at $37^{\circ} \mathrm{C}$ in a $5 \% \mathrm{CO}_{2}$ atmosphere. Plasmid transfections were performed using the Lipofectamine 2000 transfection reagent (Life Technologies, Shanghai, China) according to manufacturer's instructions when the cells reached $90 \%$ confluence. Cells were transfected with the ECHS1 siRNA plasmid or the empty pU6 vector and cultured for $24 \mathrm{~h}$. At $24 \mathrm{~h}$ post transfection, $10 \mu \mathrm{g} / \mathrm{ml}$ puromycin was added to the complete media without antibiotics in order to select for the stably transfected cell clones. The selection media was replaced every 2-4 days as necessary. Puromycin-resistant colonies started to form at $\sim 10$ days post transfection and single colonies were isolated at $~ 14$ days post transfection. The selected stably transfected cells were expanded and the examined using western blot analyses in order to confirm ECHS1 knockdown.

Western blot analysis. Cultured cells ( $~ 80 \%$ confluent) were washed three times with pre-chilled phosphate-buffered saline (PBS; Abcam), detached by trypsinization with $0.25 \%$ trypsin and then centrifuged at $25,200 \mathrm{~g}$ for $30 \mathrm{~min}$ at $4^{\circ} \mathrm{C}$. Cell pellets were first lysed with protein lysis buffer with a protease inhibitor cocktail (1:100) and sonicated on ice (5-sec sonication with intervals of $10 \mathrm{sec}$, at 100-120 W) until the lysate was clear. The cell lysates were centrifuged at 25,200 x g at $4^{\circ} \mathrm{C}$ for $15 \mathrm{~min}$ and the supernatants were stored at $-80^{\circ} \mathrm{C}$ until further use. Protein concentrations were determined using a bicinchoninic acid assay. The proteins $(10 \mu \mathrm{g})$ were subjected to $10 \%$ SDS-PAGE gel electrophoresis and transferred onto polyvinylidene difloride membranes (Millipore Corp., Billerica, MA, USA). The membranes were incubated for $1 \mathrm{~h}$ at room temperature in blocking buffer (Abcam), followed by an incubation overnight at $4^{\circ} \mathrm{C}$ with the following antibodies: Anti-ECHS1 (1:400; Abcam; cat. no. sc-1562) and anti-tubulin (1:2,000; Santa Cruz Biotechnology, Inc.; cat. no. sc-47778). The membranes were then washed with tris-buffered saline, Tween-20 (Cell Signaling Technology, Inc., Danvers, MA, USA) and Triton X-100 (Bio-Rad Laboratories, Inc., Hercules, CA, USA), and incubated with horseradish peroxidase-conjugated anti-rabbit antibody (1:8,000; ZSGB-BIO, Beijing, China; cat. no. sc-81656) for $2 \mathrm{~h}$ at $37^{\circ} \mathrm{C}$. The blots were visualized with enhanced chemiluminescence solution (Applygen Technologies, Inc., Beijing, China) and exposed to X-ray films (LAS-1000; Fujifilm, Tokyo, Japan). The density of the protein bands were assessed using TotalLab analysis software, version 2.01 (Nonlinear USA, Inc., Durham, NC, USA).

Cell proliferation assay. A Cell Counting Kit-8 (CCK-8; Bio-Rad Laboratories, Inc.) assay was used to determine the rate of cell proliferation in HCT-8 cells following ECHS1 siRNA transfection. ECHS1 siRNA and empty pU6 vector stably transfected cells as well as the parental HCT-8 cells growing in logarithmic phase were seeded into 96-well plates at a density of 5,000 cells per well (in $200 \mu$ l culture media) in quadruplicate. The CCK- 8 reagent $(10 \mu \mathrm{l})$ was added to each well and cells were then incubated at $37^{\circ} \mathrm{C}$ for $90 \mathrm{~min}$. Absorbance values were measured at $450 \mathrm{~nm}$ using a microplate reader (DNM-9606; Nanjing Perlove Medical Equipment Co., Ltd., Nanjing, China). CCK-8 assays were performed following 12, 24, 36 and $48 \mathrm{~h}$ of culture post transfection.

Cell migration assay. The Transwell ${ }^{\circledR}$ method was used in order to determine cell migration of colorectal cancer cells. Cell suspensions were prepared by trypsinizing the cultured cells, washing twice with serum-free media (Abcam), triturating the cells into single cell suspensions and then adjusting the cell concentration to $1 \times 10^{5}$ cells $/ \mathrm{ml}$. The upper chambers of the Transwell ${ }^{\circledR}$ (Santa Cruz Biotechnology, Inc.) were placed onto a 24-well plate containing $500 \mu 1$ media with 20\% FBS or chemokine (Applygen Technologies, Inc.). Cell suspension $(200 \mu \mathrm{l})$ was added to the upper chamber of the Transwell ${ }^{\circledR}$ assembly and incubated at $37^{\circ} \mathrm{C}$ in a $5 \% \mathrm{CO}_{2}$ 


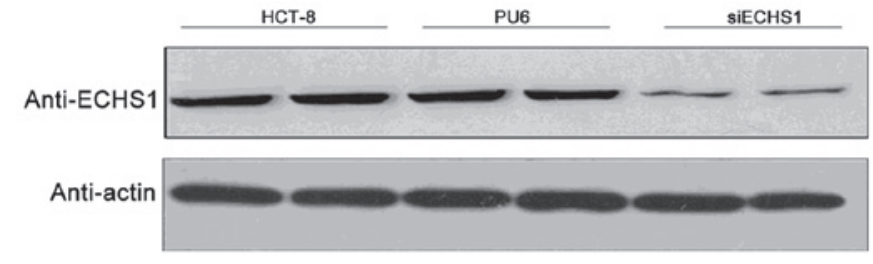

Figure 1.ECHS1 protein levels are reduced in ECHS1-silenced cells. Western blot analysis of the parental HCT-8 as well as empty PU6 vector-transfected and ECHS1-silenced cells. Results of two independent cell lines are shown. ECHS1, enoyl coenzyme A hydratase 1; siECHS1, HCT-8 cells transfected with ECHS1 siRNA; PU6, HCT-8 cells stably transfected with the empty pU6 vector.

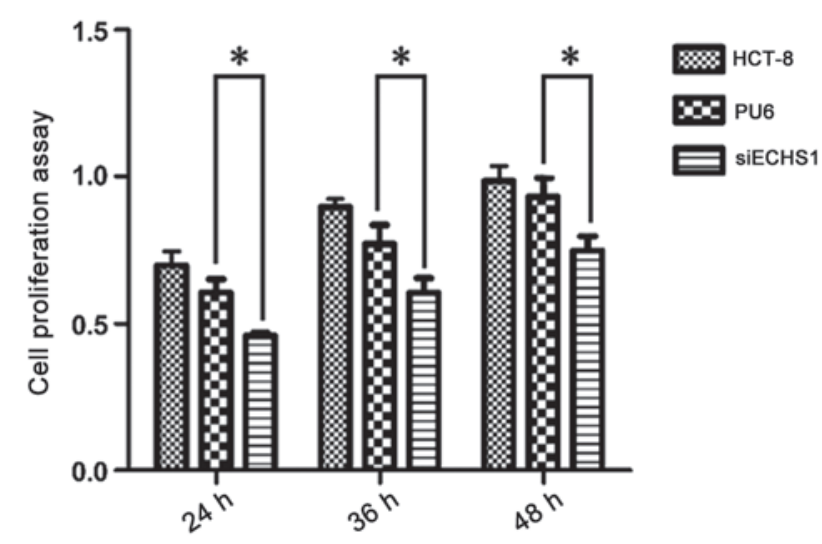

Figure 2. Cell proliferation analysis of ECHS1-silenced cells. Parental HCT-8 as well as empty PU6 vector-transfected and ECHS1-silenced cells were seeded at a density of 5,000 cells/well and cultured for 24,36 or $48 \mathrm{~h}$. Cell counting kit- 8 assays were performed at each time-point in order to estimate the number of live cells. Values are presented as the mean \pm standard deviation; " $\mathrm{P}<0.05$. ECHS1, enoyl coenzyme A hydratase 1; siECHS1, HCT-8 cells expressing ECHS1 siRNA; PU6, HCT-8 cells stably transfected with the empty pU6 vector.

atmosphere for $16 \mathrm{~h}$. The Transwell ${ }^{\circledast}$ assembly was then washed with PBS twice and fixed with pre-chilled methanol (Invitrogen Life Technologies) at $-20^{\circ} \mathrm{C}$ for $10 \mathrm{~min}$. The upper chamber of the Transwell ${ }^{\circledR}$ assembly was washed with PBS twice. The cells which remained on the top surface of upper chamber were removed using a wet cotton swab. The upper chamber was then washed with PBS three times and air-dried in an upside-down position. The chamber membrane was stained with $500 \mu \mathrm{l} /$ well $0.1 \%$ crystal violet staining solution (GE Healthcare Life Sciences, Little Chalfont, UK) at $37^{\circ} \mathrm{C}$ for $30 \mathrm{~min}$, washed with PBS three times and then air-dried. The crystal violet retained on the Transwell ${ }^{\circledR}$ was eluted with $33 \%$ acetic acid (Sigma-Aldrich, St. Louis, MO, USA) and the optical density $(\mathrm{OD})_{570 \mathrm{~nm}}$ value of the eluent was measured using a microplate reader in order to indirectly estimate the number of cells migrated through the Transwell ${ }^{\circledR}$ membrane.

Statistical analysis. GraphPad Prism 5 statistical analysis software was used for data processing (GraphPad Software, Inc., La Jolla, CA, USA). Values are presented as the mean \pm standard deviation. Differences between two sets of values were compared using the Student's $t$-test. Analyses for fitness to normal distribution and homogeneity of variance of the various experimental groups were performed. $\mathrm{P}<0.05$
$\mathbf{A}$
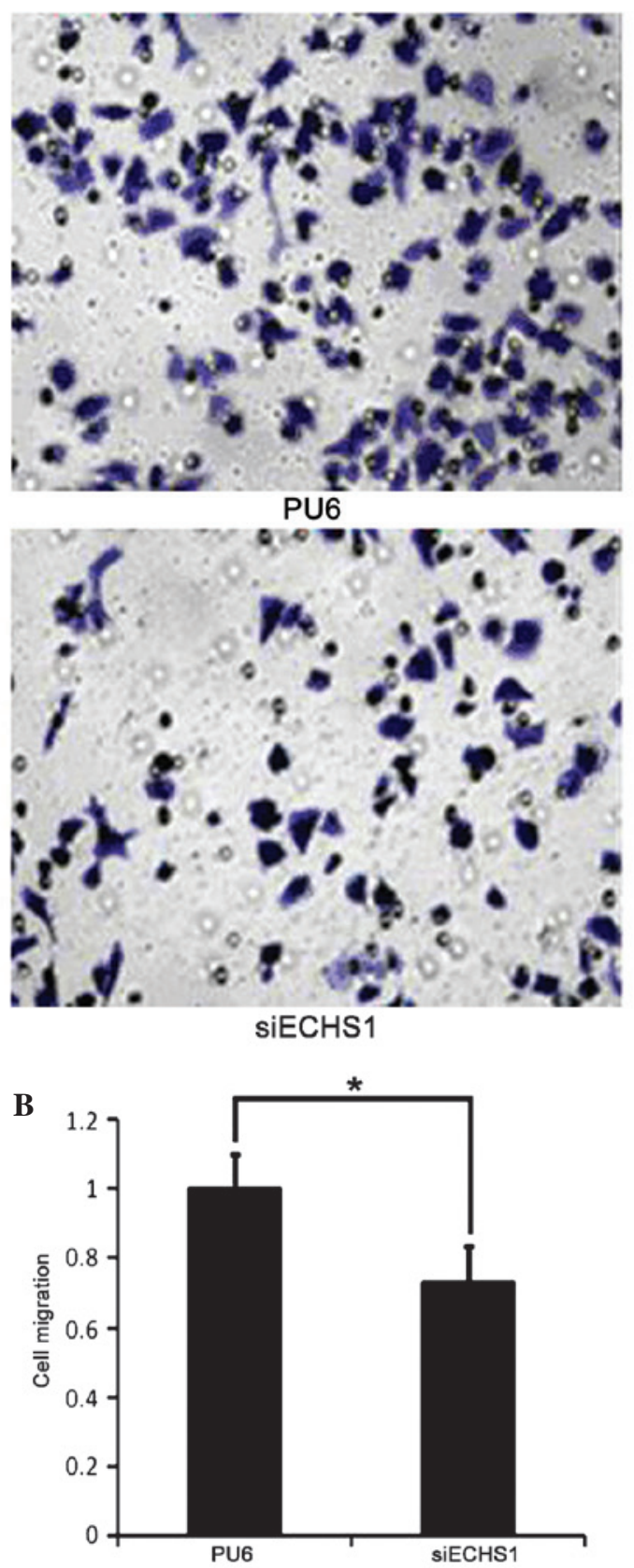

Figure 3. Cell migration analysis of siECHS1 cells. (A) Light microscopy images of crystal violet-stained PU6 or siECHS1 cells that migrated through the Transwell ${ }^{\circledast}$ membrane (magnification, $\mathrm{x} 400$ ). (B) Quantification of the migration of PU6- and siECHS1- transfected cells. Values are presented as the mean \pm standard deviation; " $\mathrm{P}<0.05$. ECHS1, enoyl coenzyme A hydratase 1; siECHS1, HCT-8 cells expressing ECHS1 siRNA; PU6, HCT-8 cells stably transfected with the empty pU6 vector.

was considered to indicate a statistically significant difference between values.

\section{Results}

Establishment of a stably transfected ECHS1-silenced cell line. In order to study the functions of ECHS1 in colorectal cancer cells, a stably transfected HCT-8 cell line expressing siRNA targeting the ECHS1 gene was established using the pU6-siECHS1 vector, which were named siECHS1 cells. In addition, HCT-8 cell lines stably transfected with the empty 

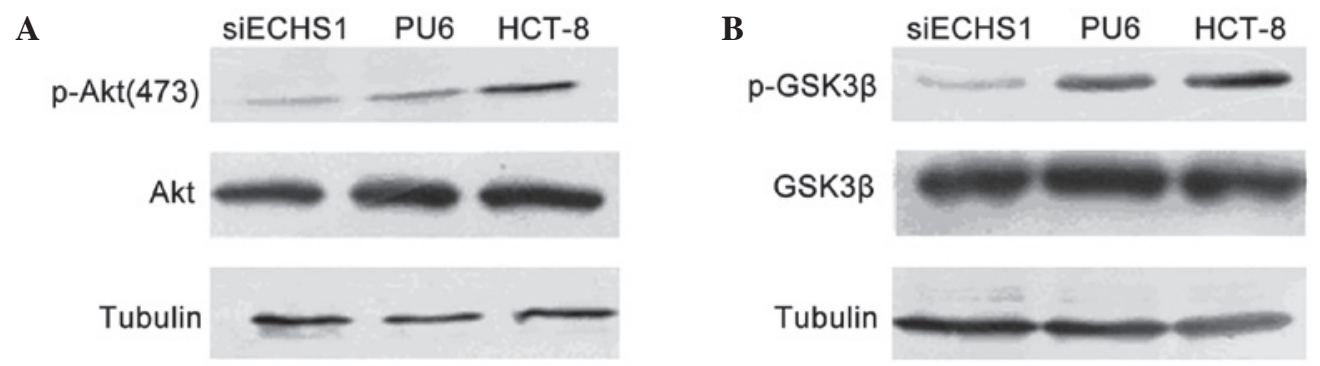

Figure 4. Effects of ECHS1 knockdown on Akt and GSK3 $\beta$ phosphorylation. Western blot analysis of (A) Akt phosphorylation at serine 473 and (B) GSK3 $\beta$ phosphorylation in siECHS1, PU6 and parental HCT-8 cells. Tubulin was used as the loading control. ECHS1, enoyl coenzyme A hydratase 1; GSK3 $\beta$, glycogen synthase kinase 3 $\beta$; siECHS1, HCT-8 cells expressing ECHS1 siRNA; PU6, HCT-8 cells stably transfected with the empty pU6 vector; p-, phosphorylated.

pU6 vector were established as controls and were named PU6 cells. As shown in Fig. 1, compared with that of the parental HCT- 8 cells, there was an $>40 \%$ decrease in ECHS1 protein expression in the siECHS1 cells, whereas no obvious decrease in ECHS1 protein levels was observed in the control PU6 cells. This therefore confirmed that stably transfected siECHS1 cells that expressed less ECHS1 protein than the parental cells were successfully established.

Cell proliferation is inhibited in siECHS1 cells. Cell proliferation profiles of siECHS1 cells were compared to those of the PU6 and parental HCT- 8 cells using CCK-8 assays. Cells were cultured for 24,36 and $48 \mathrm{~h}$ prior to assaying with CCK-8 and the number of living cells was proportional to the $\mathrm{OD}_{450 \mathrm{~nm}}$ value. The results showed that the $\mathrm{OD}_{450 \mathrm{~nm}}$ value of siECHS1 cell cultures was significantly lower compared with those of PU6 and the parental HCT-8 cells at each time-point $(\mathrm{P}<0.05)$ (Fig. 2). These results suggested that silencing of ECHS1 in HCT-8 cells inhibited cell proliferation.

Cell migration is suppressed in siECHS1 cells. The effect of ECHS1 knockdown on HCT-8 cell migration was investigated using a Transwell ${ }^{\circledR}$ assay. It was observed that fewer siECHS1 cells migrated through the transwell membrane compared with PU6 cells (Fig. 3A). Cell migration was quantified by measuring the $\mathrm{OD}_{570 \mathrm{~nm}}$ value of the eluted crystal violet stain retained on the Transwell ${ }^{\circledR}$ membrane. The results showed that there was a significant $(\sim 30 \%)$ decrease in the number of migrated cells in the siECHS1 group compared with that of the PU6 group $(\mathrm{P}<0.05$; Fig. $3 \mathrm{~B})$. These results suggested that the decrease in ECHS1 expression suppressed cell migration in HCT-8 cells.

ECHS1 knockdown decreases Akt and GSK3 $\beta$ phosphorylation in HCT- 8 cells. The phosphorylation of Akt and GSK3 $\beta$ proteins in siECHS1 cells was assessed in order to explore the roles of ECHS1 in the cell cycle regulation of colorectal cancer cells. Compared with that of the parental HCT-8 and PU6 cells, the level of Akt phosphorylation at Serine 473 was decreased in siECHS1 cells (Fig. 4A). In addition, the phosphorylation level of the Akt downstream factor GSK3 $\beta$ was found to be decreased in siECHS1 cells (Fig. 4B). These results indicated that the pro-proliferation and pro-migration functions of ECSH1 may proceed via Akt- and GSK3 $\beta$-associated signaling pathways.

\section{Discussion}

ECHS1 has been identified by proteomic or gene expression profiling in numerous types of cancer cells and patient biopsies; however, there have been a limited number of functional studies into ECHS1 $(13,14)$. The results of the present study demonstrated that proliferation and migration were inhibited in HCT-8 cells with reduced ECHS1 protein levels due to the constitutive expression of ECHS1-specific siRNA. In addition, ECHS1 knockdown suppressed Akt and GSK $\beta$ phosphorylation. These results indicated that ECHS1 was involved in colorectal cancer cell proliferation and migration, the mechanism of which may proceed via PI3K-Akt- and GSK $\beta$-associated signaling pathways. These findings were consistent with those of previous reports on the role of ECHS1 in breast, prostate and liver cancers (9); in addition, the present study showed that ECHS1 was highly expressed in colorectal cancer cells. Furthermore, a previous study demonstrated that short hairpin RNA-mediated knockdown of ECHS1 protein expression inhibited Akt activation in hepatocellular carcinoma HepG2 cells (15), which was comparable to the inhibition of Akt observed following ECHS1 knockdown in colorectal cancer HCT-8 cells in the present study. Therefore, the activation of Akt signaling may be a common mechanism for ECHS1-induced cell proliferation in cancer cells. Future studies are therefore required in order to investigate the detailed mechanism for ECHS1-mediated Akt activation. As an essential mitochondrial enzyme for fatty acid metabolism, ECHS1 expression is important for mitochondrial integrity and function (16). Since ECHS1 regulates carbohydrate synthesis and glycolysis, it may have a key role in cancer cell survival during tumor growth. In the present study, ECHS1 knockdown was found to suppress GSK3 $\beta$ phosphorylation. Notably, Akt-dependent phosphorylation of GSK3 $\beta$ was reported to regulate the Wnt signaling pathway (17); however, whether ECHS1 acts in a similar manner in colorectal cancers cells requires further exploration.

Cell migration is an important property of malignant cancer. Previous studies have established the key roles of the PI3K-Akt signaling and GSK3 $\beta$ in cell migration regulation $(18,19)$. In addition, GSK3 $\beta$ was reported to regulate the expression of miRNAs miR-96, miR-182 and miR-183, which have been shown to affect the proliferation and migration of colorectal cancer cells (20). In line with these studies, the results of the present study revealed that compared with that of PU6 cells, GSK3 $\beta$ phosphorylation and cell migration was 
decreased in siECHS1 cells. These results strongly indicated that ECHS1 regulated colorectal cancer cell migration through the GSK3 3 -associated pathways.

Numerous genes associated with metabolism also have important regulatory roles in cell proliferation and migration, as energy supply is a limiting factor for tumor growth and metastasis (21). Therefore, modulation of these metabolic genes may be a plausible therapeutic approach for cancer treatment.

In conclusion, the results of the present study showed that siRNA-mediated silencing of ECHS1 expression was able to suppress colorectal cancer cell proliferation and migration. However, it remains to be elucidated whether the decreased proliferation and migration of siECHS1 cells was due to the direct functional defects of ECHS1 or the indirect effects through other molecules. Further studies are therefore required to decipher the ECHS1-interacting factors in colorectal cancer cells in order to gain mechanistic insight into ECHS1 overexpression and oncogenesis. In addition, ECHS1 knockdown suppressed Akt and GSK $\beta$ phosphorylation, which indicated that the mechanisms of ECHS1-mediated decreased cell proliferation and migration in colorectal cancer cells may proceed via PI3K-Akt- and GSK $\beta$-associated signaling pathways. Furthermore, the present study suggested that ECHS1 may be a potential therapeutic target for colorectal cancer treatment and since ECHS1 is highly expressed in colorectal cancer cells, it may also be a candidate biomarker for colorectal cancer diagnosis.

\section{References}

1. Orannapalai N, Attawettayanon W, Kanngern S Boonpipattanapong $\mathrm{T}$ and Sangkhathat S: Predicting the occurrence of cancer-associated colorectal polyp using a metabolic risk score. Mol Clin Oncol 2: 124-128, 2014.

2. Zhang GJ, Zhou T, Liu ZL, Tian HP and Xia SS: Plasma miR-200c and miR-18a as potential biomarkers for the detection of colorectal carcinoma. Mol Clin Oncol 1: 379-384, 2013.

3. Chen G, Mao B, Pan Q, Liu Q, Xu X and Ning Y: Prediction rule for estimating advanced colorectal neoplasm risk in average-risk populations in southern Jiangsu Province. Chin J Cancer Res 26: 4-11, 2014.

4. Crncec I, Pathria P, Svinka J and Eferl R: Induction of colorectal cancer in mice and hitomorphometric evaluation of tumors. Methods Mol Biol 1267: 145-164, 2015.

5. Zhang J, Sun M, Li R, Liu S, Mao J, Huang Y, Wang B, Hou L, Ibrahim MM and Tang J: Ech1 is a potent suppressor of lymphatic metastasis in hepatocarcinoma. Biomed Pharmacother 67: $557-560,2013$.

6. Janssen U, Davis EM, Le Beau MM and Stoffel W: Human mitochondrial enoyl-CoA hydratase gene (ECHS1): structural organization and assignment to chromosome 10q26.2-q26.3. Genomics 40: 470-475, 1997.
7. Sakai C, Yamaguchi S, Sasaki M, Miyamoto Y, Matsushima Y and Goto Y: ECHS1 mutations cause combined respiratory chain deficiency resulting in Leigh syndrome. Hum Mutat 36: 232-239, 2015.

8. Zhang X, Yang J, Guo Y, Ye H, Yu C, Xu C, Xu L, Wu S, Sun W, Wei H, Gao X, Zhu Y, Qian X, Jiang Y, Li Y and He F: Functional proteomic analysis of nonalcoholic fatty liver disease in rat models: enoyl-coenzyme a hydratase down-regulation exacerbates hepatic steatosis. Hepatology 51: 1190-1199, 2010.

9. Liu X, Feng R and Du L: The role of enoyl-CoA hydratase short chain 1 and peroxiredoxin 3 in PP2-induced apoptosis in human breast cancer MCF-7 cells. FEBS Lett 584: 3185-3192, 2010.

10. Gong X, Zhu Y, Dong J, Chen J, You J, Zheng Q, Rao Z, Mao Q and Jiang J: Small hepatitis B surface antigen interacts with and modulates enoyl-coenzyme A hydratase expression in hepatoma cells. Arch Virol 158: 1065-1070, 2013.

11. Chang Y, Wang SX, Wang YB, Zhou J, Li WH, Wang N, Fang DF, Li HY, Li AL, Zhang XM and Zhang WN: ECHS1 interacts with STAT3 and negatively regulates STAT3 signaling. FEBS Lett 587: 607-613, 2013.

12. Yang G, Ma F, Zhong M, Fang L, Peng Y, Xin X, Zhong J, Zhu $\mathrm{W}$ and Zhang Y: Interleukin-11 induces the expression of matrix metalloproteinase 13 in gastric cancer $\mathrm{SCH}$ cells partly via the PI3K-AKT and JAK-STAT3 pathways. Mol Med Rep 9: 1371-1375, 2014.

13. Von Ohlen T, Luce-Fedrow A, Ortega MT, Ganta RR and Chapes SK: Identification of critical host mitochondrion-associated genes during Ehrlichia chaffeensis infections. Infect Immun 80: 3576-3586, 2012.

14. Zhu XS, Gao P, Dai YC, Xie JP, Zeng W and Lian QN: Attenuation of enoyl coenzyme A hydratase short chain 1 expression in gastric cancer cells inhibits cell proliferation and migration in vitro. Cell Mol Biol Lett 19: 576-589, 2014.

15. Zhu XS, Dai YC, Chen ZX, Xie JP, Zeng W, Lin YY and Tan QH: Knockdown of ECHS1 protein expression inhibits hepatocellular carcinoma cell proliferation via suppression of Akt activity. Crit Rev Eukaryot Gene Expr 23: 275-282, 2013.

16. Xie JP, Zhu XS, Dai YC, Yu C, Xie T and Chen ZX: Expression of enoyl coenzyme A hydratase, short chain, 1, in colorectal cancer and its association with clinicopathological characteristics. Mol Clin Oncol 2: 1081-1084, 2014.

17. Zhang L, Zhang J, Dong Y, Swain CA, Zhang Y and Xie Z: The potential dual effects of sevoflurane on AKT/GSK3beta signaling pathway. Med Gas Res 4: 5, 2014.

18. Chen L, Wei X, Hou Y, Liu X, Li S, Sun B, Liu X and Liu H: Tetramethylpyrazine analogue CXC195 protects against cerebral ischemia/reperfusion-induced apoptosis through PI3 K/Akt/GSK3 $\beta$ pathway in rats. Neurochem Int 66: 27-32, 2014.

19. Zhou RJ, Wang F, Zhang XH, Zhang JJ, Xu J, Dong W and Zou ZQ: A-eleostearic acid inhibits growth and induces apoptosis in breast cancer cells via HER2/HER3 signaling. Mol Med Rep 9: 993-998, 2014.

20. Wang R, Li Z, Guo H, Shi W, Xin Y, Chang W and Huang T: Caveolin 1 knockdown inhibits the proliferation, migration and invasion of human breast cancer BT474 cells. Mol Med Rep 9: 1723-1728, 2014

21. Yunqiao L, Vanke H, Jun X and Tangmeng G: MicroRNA-206, down-regulated in hepatocellular carcinoma, suppresses cell proliferation and promotes apoptosis. Hepatogastroenterology 61: 1302-1307, 2014. 